2001;50(2):322-328.

17. Uhde I, Toman A, Gross I, Schwanstecher C, Schwanstecher M. Identification of the potassium channel opener site on sulfonylurea receptors. J Biol Chem. 1999;274(40):28079-28082

18. Glaser B, et al. Familial hyperinsulinism caused by an activating glucokinase mutation. NEngl J Med. 1998; 338(4):226-230.
19. Stanley CA, et al. Hyperinsulinism and hyperammonemia in infants with regulatory mutations of the glutamate dehydrogenase gene. N Engl J Med. 1998; 338(19):1352-1357.

20. Otonkoski T, et al. Physical exercise-induced hypoglycemia caused by failed silencing of monocarboxylate transporter 1 in pancreatic $\beta$ cells. Am J Hum Genet. 2007;81(3):467-474.
21. Hardy OT, et al. Functional genomics of the $\beta$-cell: short-chain 3-hydroxyacyl-coenzyme A dehydrogenase regulates insulin secretion independent of $\mathrm{K}^{+}$ currents. Mol Endocrinol. 2007;21(3):765-773.

22. Li C, et al. Mechanism of hyperinsulinism in shortchain 3-hydroxyacyl-CoA dehydrogenase deficiency involves activation of glutamate dehydrogenase. J Biol Chem. 2010;285(41):31806-31818.

\title{
ApoE controls the interface linking lipids and inflammation in atherosclerosis
}

\author{
Christian Weber ${ }^{1,2}$ and Oliver Soehnlein ${ }^{1}$
}

\author{
${ }^{1}$ Institute for Cardiovascular Prevention, Ludwig-Maximilians-University Munich, Munich, Germany. ${ }^{2}$ Munich Heart Alliance, Munich, Germany.
}

\begin{abstract}
Atherosclerosis is a chronic inflammatory disease of the arterial walls that often leads to myocardial infarction and/or stroke. Hypercholesterolemia and an imbalance of peripheral leukocyte counts, leading to arterial leukocyte infiltration, are considered independent risk factors for atherosclerosis. However, in this issue of the JCI, Murphy and colleagues identify a mechanistic link between hypercholesterolemia, leukocytosis, and the subsequent development of atherosclerotic lesions in mice. These findings could pave the way for the development of novel treatment strategies to control leukocyte homeostasis and atherosclerosis.
\end{abstract}

Atherosclerosis is a chronic inflammatory disease of the arteries that increasingly threatens human health worldwide as a result of its debilitating, and sometimes lifethreatening, consequences, which include limb ischemia, myocardial infarction, and stroke. Identification of novel therapeutic targets is needed to put the brakes on the global increase in the number of cases of atherosclerosis-related diseases.

Two major factors contributing to the pathophysiology of atherosclerosis are hyperlipidemia and inflammation (1). Hyperlipidemia, in particular hypercholesterolemia, is regarded as an independent risk factor in the development of ischemic heart disease, including myocardial infarction $(1,2)$. Epidemiologic studies have established a strong correlation between elevated total cholesterol levels in serum and morbidity and mortality from myocardial infarction (2). Elevated numbers of circulating neutrophils and monocytes have also been shown to be predictive of cardiovascular events independent of serum cholesterol levels $(3,4)$. Consistent with this, recent data from mouse models has provided evidence of a direct correlation between the number of circulating and

Conflict of interest: Christian Weber and Oliver Soehnlein are stakeholders of Carolus Therapeutics Inc.

Citation for this article: J Clin Invest. 2011; 121(10):3825-3827. doi:10.1172/JCI60457. lesional neutrophils or monocytes and atherosclerotic lesion size $(5,6)$.

In this issue of the JCI, Murphy and colleagues have identified ApoE on hematopoietic stem and multipotential progenitor cells (HSPCs) as part of an ABC transporter-mediated cholesterol efflux pathway and suppressor of proliferation, providing a mechanistic link between hypercholesterolemia, leukocytosis, and the subsequent development of atherosclerotic lesions in mice (ref. 7 and Figure 1). These data have the potential to open the door to the development of novel options for treating atherosclerosis.

\section{ApoE controls HSPC proliferation and atherosclerosis development}

The most commonly used mouse models of atherosclerosis involve feeding a high-fat diet to mice lacking either ApoE or LDLR. Mice lacking ApoA1 (a major component of HDL) do not develop atherosclerosis, but do manifest hypercholesterolemia. Murphy and colleagues report that Apoe ${ }^{-/-}$mice fed a high-fat diet responded with pronounced proliferation of HSPCs, neutrophilia, and monocytosis, whereas $\mathrm{Ldlr}^{/-}$and Apoa1 $1^{-/-}$mice exhibited modest increases in these parameters, suggestive of a specific role for ApoE in suppressing HSPC proliferation (7).

Subsequent analyses showed that ApoE was expressed on the surface of HSPCs and that its expression was further upregulated after treatment with liver $\mathrm{X}$ receptor (LXR) activators (7), which have previously been shown to reduce both HSPC proliferation and atherosclerotic lesion formation $(8,9)$. Moreover, Murphy and colleagues determined that HSPCs themselves secreted ApoE, which became anchored to proteoglycans on the cell surface (7). There it was shown to interact with ABCA1, which transports excessive cholesterol from membranes to nascent HDL particles, and ABCG1, which transports cholesterol to mature HDL particles, thereby controlling intracellular cholesterol levels. The authors then showed that in HSPCs lacking ApoE, cholesterol efflux pathways were disrupted and cholesterol accumulated intracellularly, resulting in increased responsiveness to the hematopoietic growth factors IL-3 and GM-CSF (Figure 1). The net effect was an increase in HSPC proliferation, neutrophilia, and monocytosis, with accelerated atherosclerosis. Thus, a proteoglycan-bound reservoir of ApoE on HSPCs controls their proliferation and circulating leukocyte numbers.

In a therapeutic experiment, Murphy and colleagues injected Apoe ${ }^{-/}$mice with cholesterol-poor phospholipid/ApoA-I complexes as cholesterol acceptors, thereby restoring normal HSPC proliferation and reducing the expansion of circulating neutrophils and monocytes (7). All the data generated by Murphy and colleagues provide convincing evidence of a mechanistic link between hypercholesterolemia and leukocytosis, and indicate that this contributes to the development of atherosclerotic lesions in mice.

\section{Keeping the ill alliance of lipids and leukocytosis in check}

To fully appreciate the importance of the data provided in this study (7) and to be able to translate these data into the devel- 


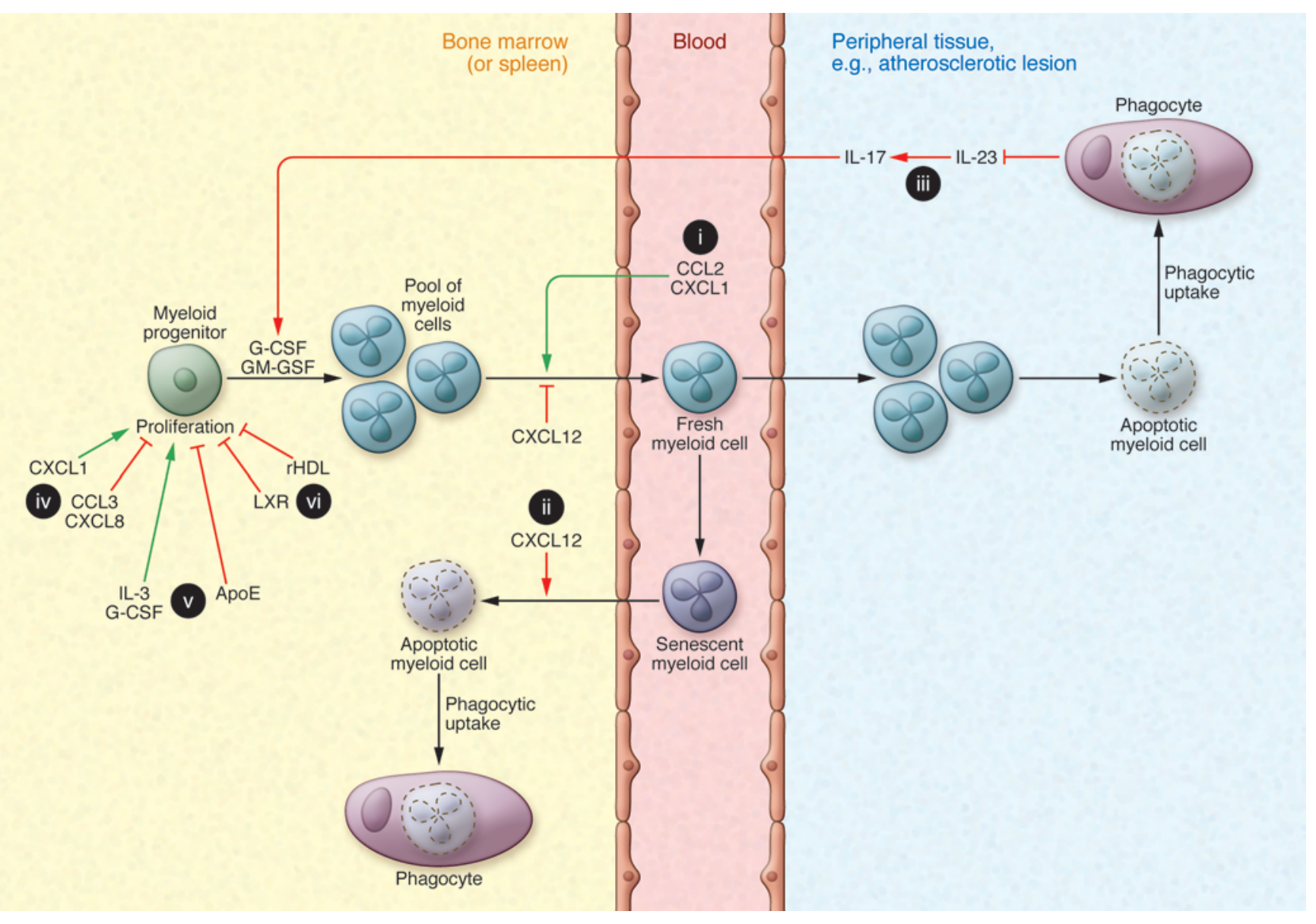

Figure 1

Chemokines and lipid metabolism synergize in the control of circulating myeloid cell numbers. Mechanisms controlling myeloid cell mobilization and clearance (i-iii), as well as mechanisms regulating proliferation of myeloid progenitor cells and means of therapeutic intervention (iv-vi), are shown. (i) Levels of plasma CCL2 and CXCL1 critically determine mobilization of myeloid cells from the bone marrow pool into the circulation, whereas CXCL12 exerts retention signals. (ii) Bone marrow CXCL12 levels control homing of senescent myeloid cells to the bone marrow. (iii) Clearance of apoptotic cells by macrophages in peripheral tissue, e.g., in atherosclerotic lesions, unleashes a negative feedback loop involving IL-23 and IL-17. (iv) CCL3 and CXCL8 exert myelosuppressive effects, which are counteracted by CXCL1. (v) Endogenous ApoE bound to proteoglycans interacts with $\mathrm{ABCA1}$ and $\mathrm{ABCG} 1$ to enhance cholesterol transportation mechanisms, thereby reducing proliferation of myeloid progenitor cells. (vi) Treatment with LXR agonists enhances expression of ApoE, ABCA1, and ABCG1, thus stimulating cholesterol efflux and dampening proliferation of myeloid progenitor cells. Similarly, cholesterol-poor phospholipid/ApoA-I complexes (rHDL) inhibit proliferation of myeloid progenitor cells. Mechanisms contributing to reduced circulating myeloid cell numbers are shown in red; mechanisms contributing to increased circulating myeloid cell numbers are shown in green; migration or differentiation pathways are shown in black.

opment of novel therapeutic interventions, several questions remain to be answered. First, how selective are these mechanisms for hypercholesterolemia-induced leukocytosis? Myeloid cell homeostasis is dysregulated in various pathological conditions, including acute inflammatory processes and neoplastic myeloproliferative disorders. In both these cases, myeloid precursor proliferation is usually increased, but the contribution of ApoE, ABCG1, and ABCA1 under these conditions is unknown. Thus, it should be tested whether interference with ApoE signaling pathways affects HSPC proliferation under conditions other than hyperlipidemia.
Second, how important are other mechanisms regulating myeloid cell homeostasis? Myeloid cell homeostasis is regulated at various levels, including cell production in the bone marrow or in alternative sites such as the spleen (10), mobilization from the bone marrow (which involves signaling via CXCR2 and CXCR4), survival in the circulation, and clearance in the bone marrow or spleen. Additionally, myeloid cell homeostasis is regulated by negative feedback loops involving IL-23 release from macrophages taking up apoptotic neutrophils and monocytes (Figure 1). Previous work has pointed to the importance of facilitated mobilization, continued prolif- eration, and prolonged life span for neutrophilia and monocytosis in hypercholesterolemia $(6,11)$. Careful dissection of the various mechanisms of leukocytosis under high-fat diet conditions therefore seems warranted.

Finally, how important is the mechanism uncovered by Murphy and colleagues in regulating monocyte accumulation in atherosclerosis? Leukocyte accumulation in an atherosclerotic lesion is determined by the rate of cell recruitment, the extent of cell survival, and the rate of egress from the lesion. The direct correlation between numbers of circulating neutrophils and monocytes and lesion size in mouse models of atherosclero- 
sis $(5,6)$ suggests that disruption of myeloid cell homeostasis plays a dominant role in the accumulation of these cells within atherosclerotic lesions. Further support for this idea is provided by observations in mice in which myeloid cell mobilization from the bone marrow and clearance from the circulation is disrupted (12-14). Knockdown of CCR 2 crucially impairs mobilization of classical monocytes from the bone marrow under inflammatory conditions (12), leading to reduced atherosclerotic lesion sizes (13). In contrast, blockade of CXCR4 reduces myeloid cell clearance from the circulation, and the ensuing neutrophilia accelerates atherosclerosis (14). Compared with single gene deletions, combined deficiency of CCL2 (a CCR2 ligand) and CX3CR1 or of CCR2 and CX3CL1 decreases atherosclerosis, a finding attributed to both attenuation of hyperlipidemia-associated blood monocytosis and accumulation of macrophages in lesions $(5,15)$. Additional blockade of receptors for CCL5 to further decrease numbers of circulating monocytes almost fully protects mice against atherosclerosis (5). Thus, while the differential rate-limiting contributions of the individual steps of mobilization, recruitment, and homeostasis remain to be clarified, combined interference with the chemokine system and lipid metabolism via cholesterol efflux pathways may be most effective for controlling leukocyte numbers in peripheral blood and subsequently in atherosclerotic lesions (Figure 1).

\section{Toward new avenues for treating atherosclerosis}

The findings of Murphy and colleagues (7) have uncovered two new candidate therapeutic targets for the treatment of atherosclerosis: the bone marrow and lipid metabolism. Interfering with leukocyte accumulation in atherosclerosis has previously focused on inhibiting leukocyte recruitment into large arteries (16). However, targeting cell adhesion molecules has thus far proven unsuccessful, and interfering with chemokines may also impair host defense. In addition, the functions of cell adhesion molecules and chemokines are sufficiently redundant to provide significant robustness to the host defense system; thus, interfering with just one molecule may not be sufficient to yield atheroprotective effects. The work of Murphy and colleagues (7) may shift attention toward the control of leukocyte production and mobilization from the bone marrow. Interestingly, chemokines may not just contribute to mobilization of myeloid cells from the bone marrow, but also synergize with ApoE in the control of HSPC proliferation (Figure 1). Several chemokines, including CCL3 and CXCL8, have been shown to suppress HSPC proliferation, and may thus potentially counteract ApoE-mediated induction of hematopoiesis (17). On the other hand, an increase in levels or an excess of CXCL1 (a ligand for CXCR2) may block the suppressive effects of other chemokines on myeloid progenitor cell growth or directly exert myeloproliferative capacities $(17,18)$.

Consistent evidence supports the hypothesis that HDL levels correlate inversely with cardiovascular risk (19). Numerous approaches to increase HDL levels exist or are in development, including directly or indirectly augmenting ApoA-I levels, mimicking the functionality of ApoA-I with mimetic peptides, and enhancing steps in the reverse cholesterol transport pathway (e.g., via activation of LXR) (19). However, because of the heterogeneity in HDL particles, the complicated pathways of HDL-mediated cholesterol flux, and the association of HDL with many proteins modifying atherosclerosis, steadystate levels of HDL cholesterol in blood poorly reflect HDL function. HDL particles can affect reverse cholesterol transport and exert other antiinflammatory effects. Despite our considerable understanding of HDL and its metabolism, none of the pharmacological agents tested so far has offered a practical and proven way to reduce cardiovascular events. Furthermore, treatment of Apoe $e^{-/-}$ mice with a viral vector encoding ApoE, which lowers total cholesterol while raising HDL, did not affect blood monocyte counts and rather limited their activation (20), which indicates that the effect of ApoE on HSPCs is likely unaffected and requires more selective or sustained intervention. Thus, the work of Murphy and colleagues (7) highlights pathways that may be useful for more specific targeting in the treatment of atherosclerosis.

\section{Acknowledgments}

The authors' research is supported by the German Research Foundation (DFG), the German Heart Foundation, the GermanIsraeli Foundation, the European Research Council, the Leducq Foundation, and the Netherlands Organisation for Scientific Research (NWO).

Address correspondence to: Christian Weber or Oliver Soehnlein, Institut für Prophylaxe und Epidemiologie der Kreislaufkrankheiten, Klinikum der Universität München, Pettenkoferstr. 9, 80336 München, Germany. Phone: 49.0.89.5160.4350; Fax: 49.0.89.5160.4352;
E-mail: christian.weber@med.uni-muenchen. de (C. Weber); oliver.soehnlein@med.unimuenchen.de (O. Soehnlein).

1. Libby P, Ridker PM, Hansson GK. Progress and challenges in translating the biology of atherosclerosis. Nature. 2011;473(7347):317-325.

2. Thomas HE Jr, Kannel WB, Dawber TR, McNamara PM. Cholesterol-phospholipid ratio in the prediction of coronary heart disease. The Framingham study. NEngl J Med. 1966;274(13):701-705.

3. Olivares R, Ducimetière P, Claude JR. Monocyte count: a risk factor for coronary heart disease? $\mathrm{Am}$ J Epidemiol. 1993;137(1):49-53.

4. Giugliano G, Brevetti G, Lanero S, Schiano V, Laurenzano E, Chiariello M. Leukocyte count in peripheral arterial disease: A simple, reliable, inexpensive approach to cardiovascular risk prediction. Atherosclerosis. 2010;210(1):288-293.

5. Combadière $\mathrm{C}$, et al. Combined inhibition of CCL2, CX3CR1, and CCR5 abrogates Ly6C(hi) and Ly6C(lo) monocytosis and almost abolishes atherosclerosis in hypercholesterolemic mice. Circulation. 2008;117(13):1649-1657.

6. Drechsler M, Megens RT, van Zandvoort M, Weber C, Soehnlein O. Hyperlipidemia-triggered neutrophilia promotes early atherosclerosis. Circulation. 2010;122(18):1837-1845.

7. Murphy AJ, et al. ApoE regulates hematopoietic stem cell proliferation, monocytosis, and monocyte accumulation in atherosclerotic lesions in mice. J Clin Invest. 2011;121(10):4138-4149.

8. Yvan-Charvet L, et al. ATP-binding cassette transporters and HDL suppress hematopoietic stem cell proliferation. Science. 2010;328(5986):1689-1693.

9. Rader DJ, Daugherty A. Translating molecular discoveries into new therapies for atherosclerosis. Nature. 2008;451(7181):904-913.

10. Swirski FK, et al. Identification of splenic reservoir monocytes and their deployment to inflammatory sites. Science. 2009;325(5940):612-616.

11. Swirski FK, et al. Ly-6Chi monocytes dominate hypercholesterolemia-associated monocytosis and give rise to macrophages in atheromata. J Clin Invest. 2007;117(1):195-205.

12. Serbina NV, Pamer EG. Monocyte emigration from bone marrow during bacterial infection requires signals mediated by chemokine receptor CCR2. Nat Immunol. 2006;7(3):311-317.

13. Boring L, Gosling J, Cleary M, Charo IF. Decreased lesion formation in CCR2 $2^{-/-}$mice reveals a role for chemokines in the initiation of atherosclerosis. Nature. 1998;394(6696):894-897.

14. Zernecke A, et al. Protective role of CXC receptor 4/ CXC ligand 12 unveils the importance of neutrophils in atherosclerosis. Circ Res. 2008;102(2):209-217.

15. Broxmeyer HE. Chemokines in hematopoiesis. Curr Opin Hematol. 2008;15(1):49-58.

16. Koenen RR, Weber C. Therapeutic targeting of chemokine interactions in atherosclerosis. Nat Rev Drug Discov. 2010;9(2):141-153.

17. Saederup N, Chan L, Lira SA, Charo IF. Fractalkine deficiency markedly reduces macrophage accumulation and atherosclerotic lesion formation in CCR2 $2^{-/}$mice: evidence for independent chemokine functions in atherogenesis. Circulation. 2008;117(13):1642-1648.

18. Broxmeyer HE, et al. Macrophage inflammatory protein (MIP)-1 beta abrogates the capacity of MIP-1 alpha to suppress myeloid progenitor cell growth. J Immunol. 1991;147(8):2586-2594.

19. Degoma EM, Rader DJ. Novel HDL-directed pharmacotherapeutic strategies. Nat Rev Cardiol. 2011; 8(5):266-277.

20. Potteaux S, et al. Suppressed monocyte recruitment drives macrophage removal from atherosclerotic plaques of Apoe $\mathrm{po}^{-/}$mice during disease regression. J Clin Invest. 2011;121(5):2025-2036. 